\title{
Ion sense of polarization of the electromagnetic wave field in the electron whistler frequency band
}

\author{
B. Lundin ${ }^{1}$ and C. Krafft ${ }^{2}$ \\ ${ }^{1}$ Institute of Terrestrial Magnetism, Ionosphere and Radiowave Propagation, Russian Academy of Sciences, Troitsk, Moscow \\ Region, 142190, Russia \\ ${ }^{2}$ Laboratoire de Physique des Gaz et des Plasmas, Université Paris-Sud, 91405 Orsay Cedex, France
}

Received: 25 June 2001 - Revised: 18 January 2002 - Accepted: 29 January 2002

\begin{abstract}
It is shown that the left-hand (or ion-type) sense of polarization can appear in the field interference pattern of two plane electron whistler waves. Moreover, it is demonstrated that the ion-type polarized wave electric fields can be accompanied by the presence at the same observation point of electron-type polarized wave magnetic fields. The registration of ion-type polarized fields with frequencies between the highest ion gyrofrequency and the electron gyrofrequency in a cold, overdense plasma is a sufficient indication for the existence of an interference wave pattern, which can typically occur near artificial or natural reflecting magnetospheric plasma regions, inside waveguides (as in helicon discharges, for example), in fields resonantly emitted by beams of charged particles or, in principle, in some self-sustained, nonlinear wave field structures. A comparison with the conventional spectral matrix data processing approach is also presented in order to facilitate the calculations of the analyzed polarization parameters.
\end{abstract}

Key words. Ionosphere (wave propagation) Radio science (waves in plasma) Space plasma physics (general or miscellaneous)

\section{Introduction}

The analysis of the polarization characteristics of wave fields based on multi-component field registrations can be an efficient tool to determine the origin of the received wave signals, as well as the location of their natural or artificial sources in space or on the Earth's surface (Olson and Samson, 1979; Lefeuvre et al., 1982; Lester and Smith, 1980; Sawada et al., 1993). At the same time, the correct interpretation of the multi-component field data recorded at a single observation point cannot be performed without additional assumptions on the actual space-time structure of the registered wave fields. For example, it was pointed out that in

Correspondence to: C. Krafft

(Catherine.Krafft@lpgp.u-psud.fr) some cases, the registered fields cannot be fitted by a plane wave field pattern, but probably correspond to the interference structure of several plane waves (Southwood and Kivelson, 1984; Lundin, 1983; Lefeuvre et al., 1985). Moreover, an unsuitable modelling of the fields' structure can lead to a reduced accuracy in the determination of the waves' arriving directions (Moriyama et al., 1979) and to ambiguities in the interpretation of the physical nature of the registered wave phenomena (Manninen and Turunen, 1999, 2000).

The direction-finding technique, using the multi-component measurements of a wave magnetic field and the reconstruction of its polarization plane, is based on the essential assumption that the recorded wave field has a plane wave structure, at least in the cases when the recovered polarization plane reveals its steady-state nature during the data processing session. In other more complex cases, the structure of the wave field can be reconstructed assuming that the registration of noncorrelated plane wave field constituents is performed. In this case, the technique, which consists in recovering the so-called wave distribution function, can be used (Storey and Lefeuvre, 1974). It is shown further that in the electron whistler frequency band (which extends over more than three orders of magnitude in a dense, cold plasma that is typical of the surrounding Earth's plasma), the trivial, necessary condition for applying both techniques, which is that the sense of polarization of the reconstructed wave field should be of electron type, can be complemented by the following nontrivial sufficient condition: the revelation of the ion sense of polarization in the data is the indication that a coherent interference pattern of electron whistler plane waves is registered. If the latter situation is realized, then the orientation of the polarization planes does not correspond to the wave vectors' directions.

Actually, we show in this paper that the ion-type polarization can appear in the natural interference pattern of two electron-type polarized waves of the same frequency, but with different projections of their polarization ellipses on the plane orthogonal to the ambient magnetic field (it is assumed that each wave has a fixed polarization plane). Moreover, it 
is found that the wave interference pattern can reveal at the same observation point opposite senses of polarization for the electric and the magnetic fields. This result is shown analytically for the case of field interference patterns of two long trains of whistler wave packets (incident and reflected), registered in the region where they overlap (near the so-called reflecting region). This result allows one to justify the possibility to observe very unusual polarization features of wave fields of the electron whistler frequency range in a space plasma where the plane wave approximation is conventionally accepted.

In the laboratory plasmas' devices, where the structure of the wave fields essentially differs from that of plane waves, due to the inherent reflection from the walls, the polarization of the wave fields is known to be much more complex. Actually, both types of field polarizations have been evidenced in the interference pattern of guided whistler waves, even in the case when the helicity of the radiating antenna was adjusted for the excitation of electron-type polarized waves only (Chen and Boswell, 1997).

The paper is organized as follows. In Sect. 2, we present a strict, general analysis that shows the possibility to register ion-type field polarization in the interference pattern of two plane waves of electron sense of polarization; then, the domains of characteristic parameters where each sense of polarization can be observed in the interference pattern are determined. In Sect. 3, considering the field pattern which results from the steady-state interference of an incident and a reflected whistler wave propagating in an inhomogeneous plasma with a density gradient orthogonal to the ambient magnetic field, we show that the electric and the magnetic fields can reveal at the same observation point opposite senses of rotation around the magnetic field lines. The analysis of the interference pattern's characteristic features for the case of two noncorrelated sources of plane waves is presented in Appendix A. The relations between the calculated polarization parameters and the conventional spectral matrix elements are presented in Appendix B. In Sect. 4, we discuss the preferential conditions of observation and the typical parameters of the field interference patterns of electron whistler waves in the Earth's plasmasphere.

\section{Polarization characteristics of the electromagnetic field}

One of the conventional approaches to the analysis of multicomponent wave field data consists in calculating the elements of the so-called spectral matrix, i.e. in determining time correlations between the quasi-monochromatic wave field components. This approach is described briefly in Appendix $\mathrm{B}$ where the characteristic polarization parameters under discussion in this section are expressed using the spectral matrix elements.

Let us write the wave field $\boldsymbol{K}(t)$ as a sum of several quasimonochromatic wave field constituents of frequency $\omega$ with constant complex polarization vectors $\boldsymbol{a}_{m}\left(\boldsymbol{a}_{m}\right.$ refers to a sin- gle plane wave or to any steady-state superposition of plane waves) and complex amplitudes $h_{m}(t)$, namely:

$$
\begin{aligned}
\boldsymbol{K}(t) & \equiv \frac{1}{2} \boldsymbol{H}(t) \exp (-i \omega t)+c . c . \\
& =\frac{1}{2} \sum_{m} \boldsymbol{a}_{m} h_{m}(t) \exp (-i \omega t)+c . c .,
\end{aligned}
$$

where $\boldsymbol{K}$ can represent the electric or magnetic components $\boldsymbol{E}$ and $\boldsymbol{B}$ of the electromagnetic wave field.

The sign of the parameter $P$ represents the sense of rotation (or sense of polarization) of the field around the unit vector $\boldsymbol{n}$ directed, for example, along the ambient magnetic field $\boldsymbol{B}_{0}$

$$
\begin{aligned}
P & \equiv\left\langle\left(\left[\boldsymbol{K}(t), \boldsymbol{K}\left(t+\frac{\pi}{2 \omega}\right)\right], \boldsymbol{n}\right)\right\rangle \\
& \equiv \frac{1}{T} \int_{0}^{T}\left(\left[\boldsymbol{K}(t), \boldsymbol{K}\left(t+\frac{\pi}{2 \omega}\right)\right], \boldsymbol{n}\right) d t,
\end{aligned}
$$

where the notations [,] and (, ) indicate vector and scalar products and the brackets \langle\rangle indicate time averaging over $T$. $P>0$ corresponds to the right-hand sense of rotation of $\boldsymbol{K}(t)$ around $\boldsymbol{n}$, which corresponds to the counterclockwise sense of rotation of its projection onto the oriented plane normal to $\boldsymbol{n}$.

Under overdense, cold plasma conditions (i.e. when the electron plasma frequency $\omega_{p}$ is greater than the electron gyrofrequency $\omega_{c}$ ), typical of the Earth's magnetosphere, only electron whistler plane waves can propagate in the frequency interval $\omega_{H}<\omega<\omega_{c}\left(\omega_{H}\right.$ is the proton gyrofrequency), with electric and magnetic field vectors rotating in the same sense as the electrons gyrate around the magnetic field lines (electron-type polarization with $P>0$ ). Nevertheless, we demonstrate here that the ion-type polarization (i.e. when $P<0$ ) can be registered in the interference pattern of two plane electron whistler waves; let us mention that nothing of the kind can be observed in the case of a completely noncorrelated set of plane waves. Then, the registration of iontype polarized waves in the electron whistler frequency band is a sufficient indication for the appearance of interferences in the wave pattern, which typically can occur near artificial or natural reflecting surfaces or inhomogeneous magnetospheric plasma regions, inside waveguides, in fields resonantly emitted by beams of charged particles or, in principle, in some self-sustained nonlinear wave field structures (compare to Lundin, et al., 1996, for a hot plasma). In these cases, the complex amplitudes of the constituents of the total registered wave field are linked together through the physical mechanisms of reflection, wave-particle interaction or other probable nonlinear processes.

In addition to the sense of polarization, it is worth estimating the field intensity $W$ localized in the plane orthogonal to $n$

$$
\begin{aligned}
W & \equiv\langle(\boldsymbol{K}(t)-\boldsymbol{n}(\boldsymbol{K}(t), \boldsymbol{n}), \boldsymbol{K}(t)-\boldsymbol{n}(\boldsymbol{K}(t), \boldsymbol{n}))\rangle \\
& =\langle([\boldsymbol{K}(t), \boldsymbol{n}],[\boldsymbol{K}(t), \boldsymbol{n}])\rangle .
\end{aligned}
$$


Let us consider in (1) the sum of two plane waves and introduce the relative amplitude $\sigma$ and phase $\delta$ of both constituents, i.e. $h_{j}=\sigma_{j} \exp \left(i \delta_{j}\right)$ and $h_{2}=\sigma h_{1} \exp (i \delta)$. The short averaging time $T$ in (2) is assumed to be sufficiently small so that $\sigma$ and $\delta$ can be considered as constant within this time interval (but with the wave frequency verifying $\omega T \gg 1)$. Since $\sigma_{j}$ and $\delta_{j}$ vary typically from one short time interval to the other, distribution functions $F\left(\sigma_{j}, \delta_{j}\right)$ can be built for a large set of short successive intervals $T_{k}$ belonging to the total field registration duration $T_{d}$ (see also Appendix A); such functions can be used to calculate the mean values of $P$ and $W$, which is equivalent to a direct time averaging over the period $T_{d}$.

Calculating the polarization parameter $P$ according to Eq. (2), one obtains

$P=\frac{1}{2}\left\langle\sigma_{1}^{2}\right\rangle\left(p_{1}+\sigma^{2} p_{2}+2 \sigma\left|p_{12}\right| \cos \varphi\right)$,

where

$\left\langle\sigma_{1}^{2}\right\rangle \equiv \frac{1}{T} \int_{0}^{T} h_{1}(t) h_{1}^{*}(t) d t$

and

$\varphi \equiv \delta+v, \cos v=\operatorname{Re}\left(p_{12} /\left|p_{12}\right|\right)$,

$\sin v=-\operatorname{Im}\left(p_{12} /\left|p_{12}\right|\right)$,

$p_{j} \equiv i\left(\left[\boldsymbol{a}_{j}, \boldsymbol{a}_{j}^{*}\right], \boldsymbol{n}\right), \quad j=1,2, \quad p_{12} \equiv i\left(\left[\boldsymbol{a}_{1}, \boldsymbol{a}_{2}^{*}\right], \boldsymbol{n}\right)$. (6)

The notations $x^{*}, \operatorname{Re}(x)$ and $\operatorname{Im}(x)$ correspond to the complex conjugate value of $x$ and to the real and the imaginary parts of $x$, respectively; $i$ is the imaginary unit. In the same manner, one can find that

$W=\frac{1}{2}\left\langle\sigma_{1}^{2}\right\rangle\left(b_{1}+\sigma^{2} b_{2}+2 \sigma\left|b_{12}\right| \cos (\varphi+\varepsilon)\right)$,

where

$\cos \varepsilon=\operatorname{Re}\left(p_{12} b_{12}^{*} /\left|p_{12} b_{12}\right|\right)$,

$\sin \varepsilon=\operatorname{Im}\left(p_{12} b_{12}^{*} /\left|p_{12} b_{12}\right|\right)$,

$b_{j} \equiv\left|\left[\boldsymbol{a}_{j}, \boldsymbol{n}\right]\right|^{2}, \quad j=1,2, \quad b_{12} \equiv\left(\left[\boldsymbol{a}_{1}, \boldsymbol{n}\right],\left[\boldsymbol{a}_{2}, \boldsymbol{n}\right]\right)$.

Let us consider the case when electromagnetic fields of both waves rotate in the same sense around $\boldsymbol{n} \| \boldsymbol{B}_{0}$ i.e. $p_{1} p_{2}>0$. $P$ can change its sign if the equation $P(\sigma)=0$ possesses real roots $\sigma$ at least in some domain of $\varphi$. Let us show that the corresponding necessary condition, namely $\cos ^{2} \varphi \geq$ $p_{1} p_{2} /\left|p_{12}\right|^{2}$, can be satisfied for some values of $\varphi$, i.e. let us prove that $\left|p_{12}\right|^{2} \geq p_{1} p_{2}$. Using the substitutions

$\boldsymbol{t}=i a_{1}, s=i a_{2}, q=\left[a_{1}, n\right], p=\left[a_{2}, n\right]$,

as well as the relations

$(\boldsymbol{t}, \boldsymbol{p})^{*}=(\boldsymbol{p}, \boldsymbol{t})=(\boldsymbol{s}, \boldsymbol{q}), \quad[\boldsymbol{p}, \boldsymbol{q}]=-\boldsymbol{n}\left(\left[\boldsymbol{a}_{1}, \boldsymbol{a}_{2}\right], \boldsymbol{n}\right),(10)$

one obtains

$$
\begin{array}{r}
\left|p_{12}\right|^{2}-p_{1} p_{2}=(\boldsymbol{t}, \boldsymbol{p})(\boldsymbol{s}, \boldsymbol{q})-(\boldsymbol{t}, \boldsymbol{q})(\boldsymbol{s}, \boldsymbol{p}) \\
\quad=([\boldsymbol{t}, \boldsymbol{s}],[\boldsymbol{p}, \boldsymbol{q}])=\left|\left(\left[\boldsymbol{a}_{1}, \boldsymbol{a}_{2}\right], \boldsymbol{n}\right)\right|^{2} \geq 0 .
\end{array}
$$

Thus, some real values of $\varphi$ exist which satisfy the inequality $\cos ^{2} \varphi \geq p_{1} p_{2} /\left|p_{12}\right|^{2}$. However, one can see that, according to (11), the interference of two plane waves of the same plasma mode and sense of polarization, arriving along the same direction (so that $\boldsymbol{a}_{1}=\beta \boldsymbol{a}_{2}$, where $\beta$ is a complex number), cannot reveal the inverse sense of polarization in the resulting wave field pattern.

When both constituents have the same sense of polarization, i.e. $p_{1} p_{2} \geq 0, P$ and $p_{2}$ (as well as $P$ and $p_{1}$ ) have opposite signs in the $(\varphi, \sigma)$ domain $G_{+}$described by

$s_{2} \arccos \left(-s_{2} \sqrt{r}\right) \leq \varphi \leq \pi+s_{2} \arccos \left(s_{2} \sqrt{r}\right)$,

$r \equiv p_{1} p_{2} /\left|p_{12}\right|^{2}>0, s_{2}=\operatorname{sign}\left(p_{2}\right)$,

where the notation $\operatorname{sign}\left(p_{2}\right)$ indicates the sign of $p_{2}$ and

$\sigma_{-}<\sigma<\sigma_{+}$,

$\sigma_{ \pm}=\left(-s_{2} \cos \varphi \pm \sqrt{\cos ^{2} \varphi-r}\right)\left|\frac{p_{12}}{p_{2}}\right|$.

When $p_{1} p_{2} \leq 0$ (i.e. $r \leq 0$ ), $P$ and $p_{2}$ have opposite signs in the domain $G_{-}$defined by (here $\sigma_{-}<0$ )

$0 \leq \varphi \leq 2 \pi, 0<\sigma<\sigma_{+}$.

Outside the domains $G_{-}$and $G_{+}$, the sense of polarization cannot be modified, i.e. $P$ has the same sign as $p_{2}\left(\operatorname{sign}(P)=s_{2}\right)$.

Thus, we proved that even in the case when two wave constituents have the same sense of polarization, their resultant interference pattern can reveal the opposite sense of field rotation when the relative phases and amplitudes of waves satisfy the conditions (12) and (13). However, the eccentricity of the resultant field's polarization ellipse, which can be estimated by the calculation of the so-called circular polarization level parameter $C P L$ (see also Appendix B)

$C P L=\frac{P}{W}$

can essentially differ from those of the constituents.

\section{Whistler waves' interference pattern near a reflecting surface}

Let us consider the steady-state field interference pattern formed by the overlapping of incident and reflected waves propagating through an inhomogeneous background plasma layer with a density gradient $\nabla n_{e}$ orthogonal to the uniform ambient magnetic field $\boldsymbol{B}_{0}$, in the case when a long incident whistler wave pulse penetrates the more dense regions from the rarefied ones; the propagation is limited to the plane defined by the vectors $\nabla n_{e}$ and $\boldsymbol{B}_{0}$. Let us assume that overdense plasma conditions are satisfied (i.e. $\omega_{p} \gg \omega_{c}$ ) so that the local dispersion of whistler waves can be described by conventional formulas for the refractive index $N$

$N^{2}(\omega, \theta)=\frac{\omega_{p}^{2}}{\omega\left(\omega_{c}|\cos \theta|-\omega\right)}$, 


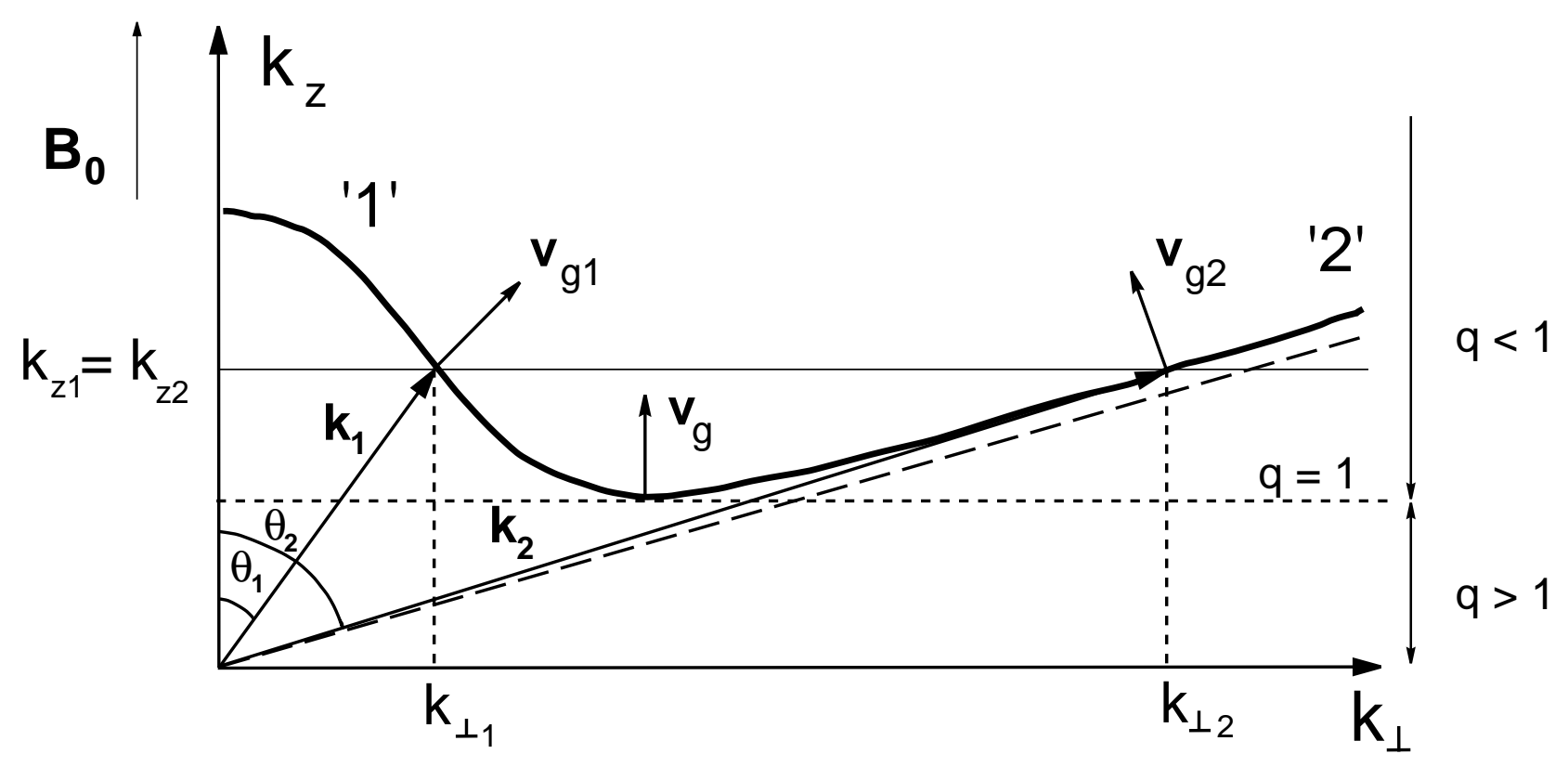

Fig. 1. Whistler dispersion curve in the plane $\left(k_{\perp}, k_{z}\right)$, at a fixed frequency $\omega<\omega_{c} / 2$. The so-called electromagnetic and quasi-electrostatic dispersion branches are labelled ' 1 ' and ' 2 ', respectively; corresponding waves are represented by their wave vectors $\boldsymbol{k}_{1}\left(k_{\perp 1}, k_{z 1}\right)$ and $\boldsymbol{k}_{2}\left(k_{\perp 2}, k_{z 2}\right)$ and their angles $\theta_{1}$ and $\theta_{2}$ with respect to the ambient magnetic field $\boldsymbol{B}_{0}$ (see also Eq. 22), respectively. Group velocity vectors are indicated as $\boldsymbol{v}_{g 1}$ and $\boldsymbol{v}_{g 2}$. The resonance cone is represented by a dashed oblique line. The reflecting surface, where the group velocity $\boldsymbol{v}_{g}$ is directed along $\boldsymbol{B}_{0}$, corresponds to $q=1$; waves are not propagating for $q>1\left(q=4 \omega_{p}^{2} \omega^{2} / \omega_{c}^{2} c^{2} k_{z}^{2}\right)$. The reflection at $q=1$ corresponds to the conversion of an incident electromagnetic whistler (branch ' 1 ') to a quasi-electrostatic whistler (branch ' 2 ') with conservation of the parallel wave numbers, $k_{z 1}=k_{z 2}$, in the presence of a gradient of plasma density across $\boldsymbol{B}_{0}$.

the polarization coefficients $\alpha_{z}^{0}, \alpha_{x}^{0}$ and $\alpha_{x}$

$\alpha_{x}^{0}=\frac{\omega|\cos \theta|-\omega_{c}}{\omega_{c}|\cos \theta|-\omega}$,

$\alpha_{z}^{0}=-\operatorname{sign}(\cos \theta) \frac{\omega \sin \theta}{\omega_{c}|\cos \theta|-\omega}$,

$\alpha_{x} \equiv \alpha_{x}^{0} \cos \theta-\alpha_{z}^{0} \sin \theta=-\operatorname{sign}(\cos \theta)$,

of the electric and magnetic fields $\boldsymbol{E}$ and $\boldsymbol{B}$

$\boldsymbol{E}=\frac{1}{2} \boldsymbol{a}_{E} E \exp (-i \omega t+i \boldsymbol{k} \cdot \boldsymbol{r})+$ c.c.,

$E=\mathcal{A} \exp (i \delta), \boldsymbol{a}_{E}=\left\{-\alpha_{x}^{0}, i,-\alpha_{z}^{0}\right\}$,

$\boldsymbol{B}=\frac{1}{2} \boldsymbol{a}_{B} B \exp (-i \omega t+i \boldsymbol{k} \cdot \boldsymbol{r})+$ c.c.,

$B=N E, \boldsymbol{a}_{B}=\left\{-i \cos \theta,-\alpha_{x}, i \sin \theta\right\}$,

the group velocity components $\boldsymbol{v}_{g z}$ and $\boldsymbol{v}_{g \perp}$ along and across $\boldsymbol{B}_{0}$, respectively,

$v_{g z}=c \frac{\left(1+\cos ^{2} \theta-2 \omega|\cos \theta| / \omega_{c}\right)}{N \cos \theta}$,

$v_{g \perp}=c \frac{\sin \theta\left(|\cos \theta|-2 \omega / \omega_{c}\right)}{N|\cos \theta|}$,

and the spectral energy density $U$ (Shafranov, 1967; Lundin, 1979)

$U=\frac{\partial\left(N^{2} \omega^{2}\right)}{\partial \omega} \frac{\left(1+\alpha_{x}^{2}\right)|E|^{2}}{16 \pi \omega}=\frac{\omega_{c}|\cos \theta|}{\left(\omega_{c}|\cos \theta|-\omega\right)} \frac{|B|^{2}}{8 \pi}$, where $\theta$ is the angle between the wave vector $\boldsymbol{k}$ and $\boldsymbol{B}_{0}$ (which is directed along the $z$-axis); $E$ and $B$ are the complex spectral amplitudes of the electric and magnetic wave fields, respectively.

One can express all the above parameters with the help of the parallel refractive index $N_{z}=c k_{z} / \omega$ (which is constant in the case of spatial gradients orthogonal to the ambient magnetic field) using the relations (see, for example, Lundin et al., 1994):

$\left|\cos \theta_{1,2}\right|=f A_{1,2}$,

$A_{1,2} \equiv \frac{2}{q}[1 \pm \sqrt{1-q}], \quad q \equiv 4 \frac{\omega_{p}^{2}}{\omega_{c}^{2}} \frac{1}{N_{z}^{2}}$,

where $f=\omega / \omega_{c}$ and

$1<A_{2} \leq 2 \leq A_{1}, \quad A_{1}+A_{2}=A_{1} A_{2}$,

$\left(A_{1}-1\right)\left(A_{2}-1\right)=1$.

The quasi-potential regime of propagation $(q \ll 1)$ corresponds to the wave dispersion branch labeled ' 2 ' on the dispersion curve $k_{z}\left(k_{\perp}, \omega\right)$ where the frequency is fixed (see Fig. 1). The two plane waves corresponding to branches ' 1 ' and ' 2 ' can propagate simultaneously only for frequencies $\omega$ less than $\omega_{c} / 2$, (more precisely, only for frequencies verifying $\omega / \omega_{c}<1 / A_{1}=[1-\sqrt{1-q}] / 2$ with $q<1$ ); plane waves are not propagating for $q>1$. The wave propagating 

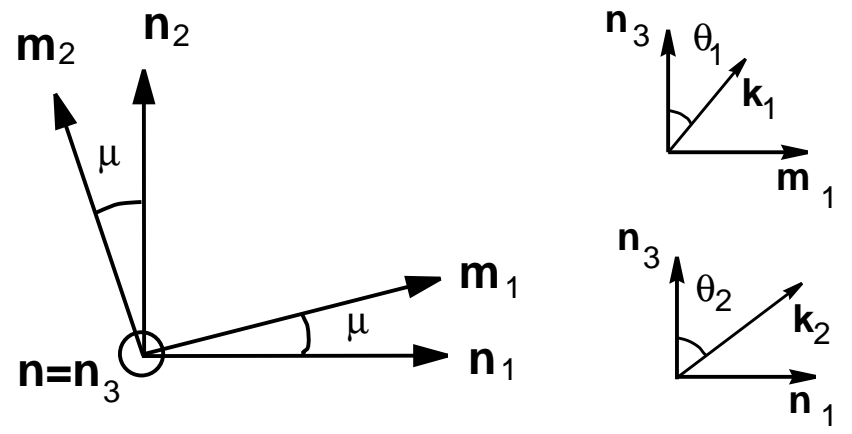

Fig. 2. Definition of the parameters governing the polarization coefficients of the incident and reflected plane waves: the plane of the two-dimensional antenna used for signal registration contains the unit vectors $\boldsymbol{n}_{1}, \boldsymbol{n}_{2}, \boldsymbol{m}_{1}$, and $\boldsymbol{m}_{2}\left(\left(\boldsymbol{n}_{1}, \boldsymbol{n}_{2}\right)=\left(\boldsymbol{m}_{1}, \boldsymbol{m}_{2}\right)=0\right.$, $\left.\left(\boldsymbol{n}_{1}, \boldsymbol{m}_{1}\right)=\cos \mu\right)$, and its oriented normal $\boldsymbol{n} \equiv \boldsymbol{n}_{3}$ is parallel to the ambient magnetic field $\boldsymbol{B}_{0}$; the wave vectors $\boldsymbol{k}_{1}$ and $\boldsymbol{k}_{2}$ of the incident and reflected waves are situated in the planes formed by the unit vectors $\boldsymbol{n}_{3}, \boldsymbol{m}_{1}$ and $\boldsymbol{n}_{3}, \boldsymbol{n}_{1}$, respectively, with $\left(\boldsymbol{k}_{1}, \boldsymbol{n}_{3}\right)=$ $k_{1} \cos \theta_{1}$ and $\left(\boldsymbol{k}_{2}, \boldsymbol{n}_{3}\right)=k_{2} \cos \theta_{2} ; \mu$ represents the angle between these planes.

in the direction of increasing plasma density and corresponding to a given branch should reflect (i.e. the perpendicular component of its group velocity $\boldsymbol{v}_{g}$ should change its sign) when $q=1$ (i.e. $\cos \theta_{1,2}=2 f$ ) and convert itself to the other branch. In this case, the reflection modifies the propagation angle, and this is the only possibility of inverting the sense of polarization in the interference pattern of incident and reflected plane waves; indeed, the waves should necessarily reveal different projections of their polarization ellipses on the plane orthogonal to the ambient magnetic field (see also Eq. 11).

The sense of polarization can be changed only if the relative amplitude $\sigma$ of the constituents (for the electric and the magnetic wave fields one notes $\sigma \equiv \sigma_{E}=\left|E_{2} / E_{1}\right|$ and $\sigma \equiv \sigma_{B}=\left|B_{2} / B_{1}\right|$, respectively) lies in the interval (see also Eq. 13)

$$
\begin{gathered}
\operatorname{sign}(r)(1-\sqrt{1-r})\left|\frac{p_{12}}{p_{2}}\right|=\sigma_{-}^{0}<\sigma \\
<\sigma_{+}^{0}=(1+\sqrt{1-r})\left|\frac{p_{12}}{p_{2}}\right|
\end{gathered}
$$

where the parameters $r, p_{2}$ and $p_{12}$ have different values for electric and magnetic field components. When $\sigma<\sigma_{-}^{0}$ or $\sigma>\sigma_{+}^{0}$, the sense of polarization cannot be inverted if $p_{1} p_{2}>0$. The parameters $r, p_{2}$ and $p_{12}$ have to be calculated for the two different cases corresponding to electric or to magnetic field registration: indeed, one should pay attention that in a magnetized plasma the polarization planes of magnetic and electric fields do not coincide, even for the case of plane waves; near the resonance cone $\left(|\cos \theta| \simeq \omega / \omega_{c}\right)$, the electric field is almost linearly polarized and almost orthogonal to the magnetic field polarization plane.

In order to obtain expressions for $\sigma_{E}$ and $\sigma_{B}$, let us consider the interference pattern of two waves propagating in two planes which cross each other along the magnetic field line $\boldsymbol{B}_{0} \| \boldsymbol{n}$ at an angle $\mu$; the wave vectors $\boldsymbol{k}_{1,2}$ and the angles $\theta_{1,2}$ verify $\cos \theta_{1,2}=\boldsymbol{k}_{1,2} \cdot \boldsymbol{n} / k_{1,2}<0$ (see also Fig. 2 for the definition of the angles $\theta_{1}, \theta_{2}$ and $\mu$ ). Then the magnetic field polarization vectors $\boldsymbol{a}_{B 1,2}$ lying on the planes orthogonal to the corresponding $\boldsymbol{k}_{1,2}$ are given by (see also Eq. 17)

$\boldsymbol{a}_{B 1}=i\left(\boldsymbol{n}_{3} \sin \theta_{1}-\boldsymbol{m}_{1} \cos \theta_{1}+i \alpha_{x 1} \boldsymbol{m}_{2}\right)$,

$\boldsymbol{a}_{B 2}=i\left(\boldsymbol{n}_{3} \sin \theta_{2}-\boldsymbol{n}_{1} \cos \theta_{2}+i \alpha_{x 2} \boldsymbol{n}_{2}\right)$,

with

$\boldsymbol{m}_{1}=\boldsymbol{n}_{1} \cos \mu+\boldsymbol{n}_{2} \sin \mu, \quad \boldsymbol{m}_{2}=\boldsymbol{n}_{2} \cos \mu-\boldsymbol{n}_{1} \sin \mu$,

where the unit vectors $\boldsymbol{n}_{1}$ and $\boldsymbol{n}_{2}$ lie on a plane orthogonal to the unit vector $\boldsymbol{n}_{3} \equiv \boldsymbol{n}$. The corresponding parameters $p_{j}, b_{j}$ and $\left|p_{12}\right|$ (see Eqs. 6 and 8) can be written as

$$
\begin{aligned}
p_{j}= & -2 \alpha_{x j} \cos \theta_{j}, \quad b_{j}=\alpha_{x j}^{2}+\cos ^{2} \theta_{j}, \quad p_{1} p_{2}>0, \\
\left|p_{12}\right|^{2}= & {\left[\left(\alpha_{x 1} \cos \theta_{2}+\alpha_{x 2} \cos \theta_{1}\right)^{2} \cos ^{2} \mu\right.} \\
+ & \left.\left(\cos \theta_{1} \cos \theta_{2}+\alpha_{x 1} \alpha_{x 2}\right)^{2} \sin ^{2} \mu\right] .
\end{aligned}
$$

Similarly, one can write the electric field polarization vectors $\boldsymbol{a}_{E 1,2}$ of the same plane wave constituents as (see also Eq. 17)

$\boldsymbol{a}_{E 1}=i\left(\boldsymbol{n}_{3} \alpha_{z 1}^{0}+i \boldsymbol{m}_{1} \alpha_{x 1}^{0}+\boldsymbol{m}_{2}\right)$,

$\boldsymbol{a}_{E 2}=i\left(\boldsymbol{n}_{3} \alpha_{z 2}^{0}+i \boldsymbol{n}_{1} \alpha_{x 2}^{0}+\boldsymbol{n}_{2}\right)$,

so that

$$
\begin{aligned}
& p_{j}=-2 \alpha_{x j}^{0}, \quad b_{j}=1+\left(\alpha_{x j}^{0}\right)^{2}, \quad p_{1} p_{2}>0, \\
& \left|p_{12}\right|^{2}=\left[\left(\alpha_{x 1}^{0}+\alpha_{x 2}^{0}\right)^{2} \cos ^{2} \mu\right. \\
& \left.+\left(1+\alpha_{x 1}^{0} \alpha_{x 2}^{0}\right)^{2} \sin ^{2} \mu\right] .
\end{aligned}
$$

Let us now consider the propagation in a common plane (i.e. $\mu=0, \boldsymbol{n} \| \boldsymbol{B}_{0}$ ) of two plane waves corresponding to the different branches ' 1 ' and ' 2 ', as mentioned above (see also Fig. 3 for a schematic representation of the waves' reflection). Then Eq. (29) becomes

$\left|p_{12}\right|=\left|\cos \theta_{2}\right|+\left|\cos \theta_{1}\right|, \quad r=q,\left|\frac{p_{12}}{p_{2}}\right|=\frac{A_{1}}{2}$,

and thus, Eq. (24) can be written as

$\sigma_{-}^{0}=1<\sigma_{B}<\sigma_{+}^{0}=A_{1}-1$.

In order to express the amplitude of the wave before and after the reflection, let us use the energy flux conservation law along the direction of the plasma density gradient, i.e. across the magnetic field $\boldsymbol{B}_{0}$. The equality of the incident and the reflected energy fluxes (see Eq. 21) at some perpendicular (i.e. across $\boldsymbol{B}_{0}$ ) distance from the reflecting surface provides the following relation (the flux cross section parallel to $\boldsymbol{B}_{0}$ is the same for the incident and the reflected whistler beams)

$\left|v_{g \perp 1}\right| U_{1}=\left|v_{g \perp 2}\right| U_{2}$, 


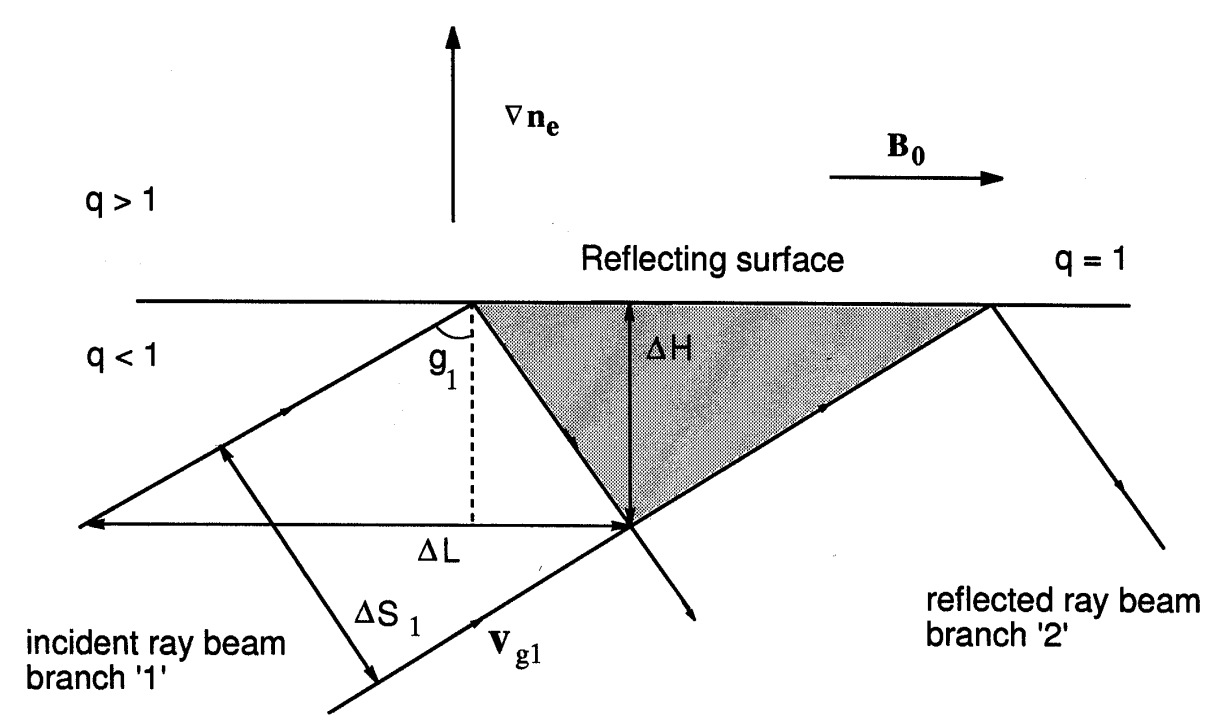

Fig. 3. Outlines of the reflection process showing the conversion of the quasi-longitudinal electromagnetic whistler branch ' 1 ' (incident ray beam) to the quasi-resonant electrostatic whistler branch '2' (reflected ray beam), respectively, (see also Fig. 1); the existence of a plasma density gradient $\nabla n_{e}$ across the ambient magnetic field $\boldsymbol{B}_{0}$ provides geometrical optics reflection of the incident ray beam at the reflecting surface where $q=1$ (see also Eq. 22). The region represented in gray is the interference zone between the incident and the reflected waves. The distance $\Delta \mathrm{H}$ represents the extension of the wave field interference pattern across the ambient magnetic field, whereas $\Delta \mathrm{L}$ and $\Delta \mathrm{S}_{1}=\Delta \mathrm{L} \cos \mathrm{g}_{1}$ are the cross sections of the incident ray beam along $\boldsymbol{B}_{0}$ and transverse to the incident group velocity $\boldsymbol{v}_{g 1}$, respectively.

leading to

$\sigma_{B}=\left|\frac{B_{2}}{B_{1}}\right|=\sqrt{\left|\frac{\sin \theta_{1}}{\sin \theta_{2}}\right|}<1$.

Thus, it is impossible to satisfy the relation $\sigma_{-}^{0}=1<\sigma_{B}$ Eq. (35): for the magnetic field components, the ion sense of polarization cannot be observed in the wave interference pattern of the two plane whistler mode branches near the reflecting surface. However, a similar conclusion is not obvious in what concerns the electric field, due to the noncoincidence of the polarization planes of electric (18) and magnetic (19) wave field components.

For the interference pattern registered by a multicomponent electric field detector (actually $\left|E_{j}\right|$ is the modulus of the electric field component transverse to $\boldsymbol{k}_{j}$ ), one can derive from Eq. (36) that

$\sigma_{E}=\left|\frac{E_{2}}{E_{1}}\right|=\sqrt{\left|\frac{\sin \theta_{1}}{\sin \theta_{2}}\right|} \frac{\cos \theta_{2}}{\cos \theta_{1}}<1$.

However, the condition similar to Eq. (35) is now written as

$$
\begin{aligned}
\sigma_{-}^{0}=\left|\frac{p_{12}}{p_{2}}\right|(1-\sqrt{1-r})<\sigma_{E} \\
<\sigma_{+}^{0}=\left|\frac{p_{12}}{p_{2}}\right|(1+\sqrt{1-r}),
\end{aligned}
$$

which can be expressed using Eqs. (32)-(33) and $p_{1} / p_{2}<1$ as

$$
\left(\frac{A_{2}}{A_{1}}\right)^{2}\left(\frac{1-A_{1} f^{2}}{1-A_{2} f^{2}}\right)=\frac{p_{1}}{p_{2}}=\sigma_{-}^{0}<\sigma_{E}<\sigma_{+}^{0}=1
$$

with

$$
\sigma_{E}=\frac{A_{2}}{A_{1}}\left(\frac{1-A_{1}^{2} f^{2}}{1-A_{2}^{2} f^{2}}\right)^{1 / 4}<1 .
$$

The conditions (40) were analyzed numerically for different parameters $q$ and $f$ (see Eq. 22). Figure 4a shows the contours of constant levels of $\sigma_{E}$ as a function of $q$ and $f$ in the validity range of Eq. (40).

The corresponding relative phase should satisfy the inequality (see Eq. 4)

$\cos \varphi<-\frac{\left(p_{1}+\sigma_{E}^{2} p_{2}\right)}{2 \sigma_{E}\left|p_{12}\right|}$,

i.e. for $p_{1}, p_{2}>0$ :

$\frac{p_{1}+\sigma_{E}^{2} p_{2}}{2 \sigma_{E}\left|p_{12}\right|}<|\cos \varphi|<1, \quad \cos \varphi<0$.

Let us prove the inequality (42) for $\sigma_{E}$ satisfying Eq. (40) at least at one observation point located at some distance $\Delta R$ from the reflecting surface; then, we will have shown that the electric field registered in the interference pattern of the two whistlers can be ion-type polarized. In order to demonstrate this, let us trace the variation of the mutual phase difference as a function of the transverse distance $\Delta R$ from the reflecting surface

$$
\begin{aligned}
& \left(k_{\perp 2}-k_{\perp 1}\right) \Delta R=\frac{\sqrt{1-q}}{q} \cdot\left(4\left(k_{z} \Delta R\right)\right) \\
& \cdot\left(\sqrt{A_{2}^{2} f^{2}\left(1-A_{1}^{2} f^{2}\right)}+\sqrt{A_{1}^{2} f^{2}\left(1-A_{2}^{2} f^{2}\right)}\right)^{-1},
\end{aligned}
$$


(a) $\sigma_{E}$

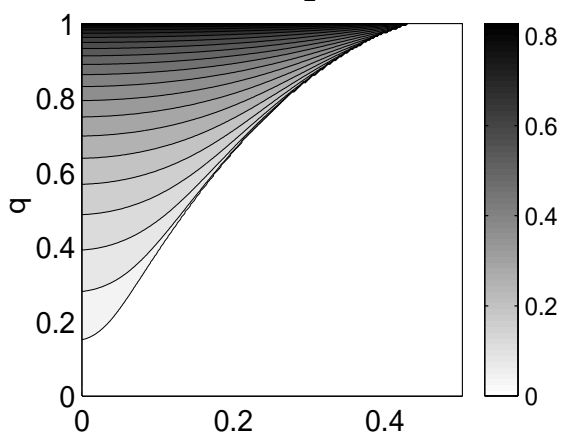

(c) $|<\mathrm{CPL}>|$

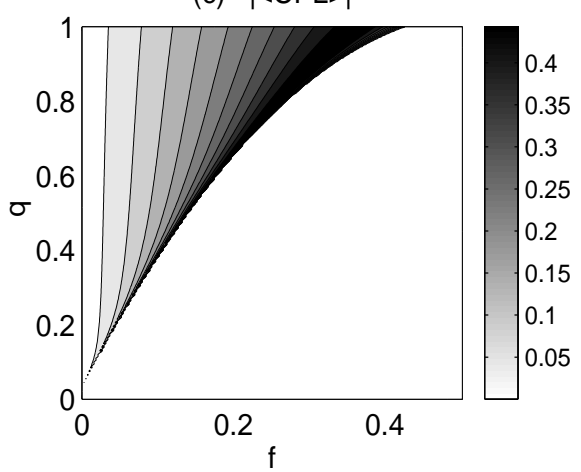

(b) $\mathrm{d}$

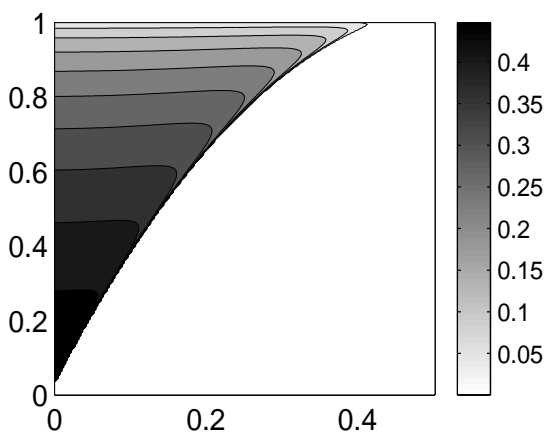

(d) $\left(\mathrm{k}_{\mathrm{z}} \Delta \mathrm{L}\right)_{\text {th }}$

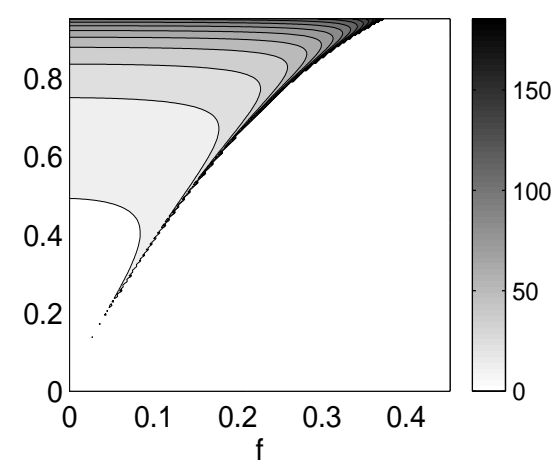

Fig. 4. Contours of constant levels in the $(q, f)$ regions compatible with the appearance of the ion sense of polarization in the field interference pattern of two plane whistlers, for different parameters characteristic of the waves' polarization: (a) electric field ratio $\sigma_{E}$ $=\left|E_{2} / E_{1}\right|$ (see Eq. 41); (b) relative plasma volume $d_{i}$ (see Eq. 45) filled by ion-type polarized electric fields; (c) absolute value of the circular polarization level $|\langle C P L\rangle|$ averaged over the regions containing ion-type polarized fields (see Eq. 49); (d) minimum threshold $\left(k_{z} \Delta \mathrm{L}\right)_{t h}$ of the phase $k_{z} \Delta \mathrm{L}$ (see Eq. 51), where $k_{z}$ is the parallel wave number of the waves and $\Delta \mathrm{L}$ the cross section of the whistler ray beam along the ambient magnetic field. In all pictures, the actual values of the parameters $\sigma_{E}, d_{i},|\langle C P L\rangle|$ and $\left(k_{z} \Delta \mathrm{L}\right)_{t h}$ have been set to zero for $(q, f)$ values where no ion-type field polarization was revealed. where $k_{\perp 1,2}$ are the perpendicular components of the wave vectors $\boldsymbol{k}_{1,2}$. Then, the following consideration can be provided : let us consider some value of $\sigma_{E}$ satisfying the conditions (40), for example, when $q \simeq 0.64$ and $f \simeq 0.1$, i.e. when $\left(k_{\perp 2}-k_{\perp 1}\right) \Delta R \simeq 6.2\left(k_{z} \Delta R\right)$. Let us assume for simplicity that the value $q \simeq 0.64$ is reached at the distance $\Delta R \equiv \Delta R_{0.64}$ and that the plasma density is homogeneous beyond this limit. Then, at $\Delta R>\Delta R_{0.64}, \sigma_{E}$ keeps a constant value, namely $\sigma_{E}=\left(\sigma_{E}\right)_{0.64}$; however, the phase difference $\left(k_{\perp 2}-k_{\perp 1}\right) \Delta R$ continues to grow so that once Eq. (42) should be satisfied, which should result in the appearance of the ion sense of polarization in the interference pattern of the two whistler waves.

Thus, we proved that the electric field measurable in the resulting interference pattern of an incident and a reflected whistler wave (reflection with conversion of dispersion branches) can reveal the ion sense of polarization. At the same time, a remarkable effect is demonstrated: the electric and the magnetic fields in the electron whistler frequency range can rotate in opposite senses around the ambient magnetic field; namely, the magnetic wave field rotates in the same sense as an electron, while the electric field can reveal the ion sense of rotation at the same points of the interference pattern.

Let us calculate the ratio $d_{i}$ of the size of the space region where the ion sense of polarization can be observed for any eccentricity of the polarization ellipse (for the case $p_{1}, p_{2}>$ $0)$ to the size of the space region corresponding to a phase variation of $2 \pi$; since the former size is proportional to the size $2 \Phi$ of the $\varphi$-interval determined by Eq. (42), one has

$d_{i}=\frac{\Phi}{\pi}, \quad \Phi=\arccos \left(\left|p_{1}+\sigma^{2} p_{2}\right| / 2 \sigma\left|p_{12}\right|\right)$.

Figure $4 \mathrm{~b}$ shows the contours of the constant levels of $d_{i}$ as a function of $f$ and $q$ in the validity range of Eq. (40). One can see that the highest values of the relative volumes filled by ion-type polarized electric fields $\left(d_{i}\right.$ is always less than 50\%) correspond to the domain of low frequencies $f$ and small parameters $q$, when one wave (here, the quasi-resonant wave labelled ' 2 ' on Figs. 1 and 3) reveals an essential electric field component along its wave vector (see also Fig. 4a). Ion-type polarized fields appear, in general, in the regions where the polarization vectors of the interfering whistler plane waves are the most different from each other; this is not the case for regions just near the reflecting surface where $q \simeq 1$ and where both incident and reflected waves are similar to each other.

In order to clarify the field structure revealed by the interference pattern, let us calculate the circular polarization level $C P L$ in the plane orthogonal to $\boldsymbol{B}_{0}$, averaged over the space region with ion-type polarized fields

$\langle C P L\rangle=\frac{\langle P\rangle}{\langle W\rangle}$.

Averaging on $\varphi$ while keeping all other parameters constant, 
one can find that

$$
\begin{gathered}
\langle P\rangle=\frac{1}{2} \sigma_{1}^{2}\left\{p_{1}+\sigma^{2} p_{2}-2 \sigma\left|p_{12}\right| \frac{\sqrt{1-\cos ^{2} \Phi}}{\Phi}\right\}, \\
\langle W\rangle=\frac{1}{2} \sigma_{1}^{2}\left\{b_{1}+\sigma^{2} b_{2}-\sigma \frac{\left(p_{1} b_{2}+p_{2} b_{1}\right)}{\left|p_{12}\right|}\right. \\
\left.\cdot \frac{\sqrt{1-\cos ^{2} \Phi}}{\Phi}\right\},
\end{gathered}
$$

where we used Eqs. (8) and (A11) of Appendix A; finally, we obtain

$$
\begin{aligned}
& \langle C P L\rangle=\widehat{C P L}[(1-\tan (\Phi) / \Phi) \\
& \left.\cdot\left(1-\widehat{C P L}\left(\left(p_{1} b_{2}+p_{2} b_{1}\right) / 2\left|p_{12}\right|^{2}\right) \tan (\Phi) / \Phi\right)^{-1}\right]
\end{aligned}
$$

with

$\widehat{C P L}=\left(p_{1}+\sigma^{2} p_{2}\right) /\left(b_{1}+\sigma^{2} b_{2}\right)$.

$\widehat{C P L}$ corresponds to the value of the circular polarization level averaged over all the space. Figure $4 \mathrm{c}$ shows the constant contour levels of $|\langle C P L\rangle|$ as a function of $f$ and $q$, in the validity range of Eq. (40). One can see that the domain of small values of both frequency $f$ and parameter $q$ is characterized by resultant electric fields with elongated polarization ellipses on the plane orthogonal to the ambient magnetic field.

For the case of a steady-state interference pattern as discussed above, the spectral matrix (see Appendix B) calculated at each observation point is singular and possesses a single, nonvanishing eigen number. The corresponding complex eigen vector calculated for the magnetic wave field, for example, and the apparent wave vector (i.e. the vector orthogonal to the magnetic field polarization plane) are not connected with the polarization plane and the wave vector of any plane wave. The direction of the apparent wave vector oscillates while the observer moves off the reflecting surface and, here in particular, its projection on the ambient magnetic field conserves its sign. It is not the case for the oriented normal to the electric field polarization plane: its projection oscillates with large angular amplitudes so that the sign of the projection to the ambient magnetic field can be opposite in different space regions. This is the actual "mechanism" of appearance of opposite polarization senses within the interference pattern.

\section{Discussion and conclusion}

In the previous section it was shown that the ion sense of polarization can appear in the interference pattern of two plane waves even when both of them are electron-type polarized. Moreover, the electric and the magnetic wave field components can reveal opposite senses of polarization at the same registration point. Then, one should not be surprised that for the complex field structures typical of waveguide modes in laboratory devices, the sense of polarization of the fields in the vicinity of the plasma chamber's axis can be opposite to that of the fields located near the walls of the tube filled by the plasma. The guided wave field is a typical example of a steady-state interference pattern: in the case of a cold plasma, it can be represented as a sum of two waves (cylindrical or not depending on the waveguide cross section) in order to satisfy the boundary conditions; then, the polarization characteristics of the total field (sense of field rotation and eccentricity of the ellipse) can be expressed with the help of the plane waves' linear polarization coefficients where, for the same parallel wave number, the relevant different transverse wave numbers (represented in general by complex values imposed by boundary conditions) are substituted. As an example, for typical waveguide modes excited in helicon discharges by antennas with spatial right- or left-hand helicities, the sense of polarization of the magnetic field near the waveguide axis is of electron or ion type, respectively, (Shoji et al., 1993). In another case, as shown on the Fig. 3 of Chen and Boswell (1997), where the magnetic field profile is characterized by one node located at some distance from the tube axis, the wave field region with ion sense of polarization (close to the walls) represents around $50 \%$ of the total tube volume (case of a radiating antenna with right-hand helicity).

Let us discuss in more detail the case of the surrounding Earth's plasma where the plane wave approximation is conventionally accepted. At least two qualitatively different cases of field interference pattern registration can be analyzed. First, considering a long whistler pulse, interferences can occur in the regions near the artificial or the natural reflecting surfaces where the incident and the reflected waves overlap. In the case of a two-dimensional propagation in the plane containing both the background magnetic field $\boldsymbol{B}_{0}$ and the density gradient $\nabla n_{e}$, the wave reflection should correspond to the conversion of whistler waves from the quasi-longitudinal to the quasi-resonant propagation regimes (or vice versa; otherwise, the necessary condition (11) is not satisfied) and this can happen in the space region with decreasing $\omega_{c} / \omega_{p}$ along the ray path. Then, under steady-state conditions, each space region reveals different polarization states, depending on the spatial variation of the waves' phases differences. As an example, for the parameters $q=0.64$ and $f=0.1$ (see also the previous section and Fig. 4b), the relative volume $d_{i}$ (45) of space filled by fields with the ion sense of polarization approaches $35 \%$. Actually, it is more easy to register ion-type polarized electric fields in the domain of small values of $f$ and $q$ where, however, the eccentricity of the polarization ellipses is rather high.

Actually, the necessity to dispose of, for physical analysis, a sufficiently extended overlapping region between incident and reflected waves (i.e. $\Delta k_{\perp} \Delta R>2 \pi$ in Eq. 44) imposes a lower limit to the width of the parallel ray beam formed by the long electromagnetic pulse. This condition can be written as (see also Fig. 3)

$$
\Delta k_{\perp} \Delta \mathrm{L}>2 \pi\left(\left|\frac{v_{g z}}{v_{g \perp}}\right|_{1}+\left|\frac{v_{g z}}{v_{g \perp}}\right|_{2}\right) \equiv\left(k_{z} \Delta \mathrm{L}\right)_{t h},
$$


where $v_{g z}$ and $v_{g \perp}$ are defined by Eq. (20) and $\Delta \mathrm{L}$ is the cross section of the beam along the ambient magnetic field.

Estimating Eq. (51) at the point $(q, f)=(0.64,0.1)$ leads to $\left(k_{z} \Delta \mathrm{L}\right)_{t h}=18$ (see also the previous section and Eq. 44 ). For such a wide beam of long electromagnetic pulse, the extension $\Delta \mathrm{H}$ of the field interference pattern across the ambient magnetic field verifies $\Delta k_{\perp} \Delta \mathrm{H}>2 \pi$; a similar condition can be written for the cross section $\Delta \mathrm{S}_{i}$ of the ray beam $i(i=1,2)$, using $\Delta \mathrm{S}_{i}=\Delta \mathrm{L} \cos g_{i}$ and $\tan g_{i}=\left|v_{g z} / v_{g \perp}\right|_{i}$ (see also Fig. 3). Figure $4 d$ represents the contours of constant levels of $\left(k_{z} \Delta \mathrm{L}\right)_{t h}$ as a function of $(q, f)$ values compatible with the occurrence of the ion-type polarization in the interference pattern. One can see that, for low frequency quasi-electrostatic whistler wave pulses, i.e. when $q$ and $f$ are both small, the condition (51) is not difficult to fulfill; thus, this domain of $(q, f)$ is preferential for the observation of unusual polarization features of whistler electric fields.

The reflection with conversion occurs typically for lightning generated whistler waves when they propagate upward in a smoothly inhomogeneous plasmaspheric plasma. The reflection with conversion to the quasi-resonant propagation regime (i.e. the change, for the same upward orientation of the wave vector, from increasing to decreasing $L$-shells along the ray path) typically takes place before the whistler wave packets can reach the plasmapause where the minimum of $\omega_{c} / \omega_{p}$ is realized. In this case, however, at least one condition (namely, small $f$ ) that favors the observations of the inverse sense of polarization is violated: typically the reflection occurs when the wave frequency is comparable with, but not much less than, the value of the local electron gyrofrequency.

The other conditions of registration are typical of waves originating from different distant sources of noncoherent nature. Then, one can reasonably assume that the phases of the wave packets arriving at the receiver are not correlated so that their mutual differences are uniformly distributed over the interval $[0,2 \pi]$ during the long total observation time $T_{d}$. In this case, different polarization states can be registered during shorter time intervals when the variation of phases is negligible; the probability to register ion-type polarization in this case can be estimated using Eqs. (A6)-(A7) of Appendix A.

One should pay attention to the possible comparatively small intensity of the wave field of the inverse sense of polarization, which arises from the interference of plane wave constituents of the same sense of polarization. Indeed, according to Eq. (4), the inverse sense of polarization can appear only if the cross field term proportional to $\left|p_{12}\right|$ dominates both the terms proportional to $p_{1}$ and $p_{2}$. However, it is not surprising that, for the same value of the phase difference $\varphi$ as in Eq. (4), the cross term in Eq. (7) can reduce the value of the total field intensity. Quantitatively, for the case of two noncorrelated sources (see Appendix A), the probability $d_{2}$ to observe the field with the inverse sense of polarization is greater than the relative intensity of the wave field $W_{2}$ with inverse sense of polarization, i.e. $d_{2}>W_{2}$ (see Eq. A12).

Thus, we demonstrate in this paper that the sense of polarization of the nonplane wave field corresponding to some interference pattern of two plane waves can be opposite to that of each wave constituent. It is shown that the ion and the electron senses of polarization can be both revealed inside the coherent mixture of plane electron whistler waves; moreover, the registration of the ion sense of polarization in an overdense, cold plasma certainly indicates the existence of a coherent wave interference pattern. One should pay attention that the "revelation of wave coherence" in the registration pattern, i.e. the actual observation of interferences, essentially depends on the inherent phase scattering time of the arriving wave packets, as well as on the receivers' frequency filters' bandwidths (which are inversely proportional to the filters recovering times or quality factors of the filters); the same remark is applicable to the width of the analogical or digital filters used in the subsequent data processing.

Nevertheless, there are some cases when a persistent phase coherence naturally appears, corresponding, for example, to the interference of waves near reflecting surfaces, inside waveguides and in the field patterns excited by the resonant emission of charged particles' beams. It follows from the fact that, at least for wave frequencies less than half of the electron gyrofrequency, the electron whistler mode in an overdense plasma $\left(\omega_{p}>\omega_{c}\right)$ is characterized by the existence of two plane waves of the same frequency and of the same parallel wave number which propagate at different angles with respect to the ambient magnetic field (and thus, which have essentially different polarization vectors, which is indispensable for satisfying condition (11) which is necessary for the inversion of the polarization sense). Then, the registration of the ion sense of polarization in the interference pattern of two whistlers should not be, in principle, a very surprising phenomenon; however, the eccentricity of the polarization ellipse of the total field can differ noticeably from that of each plane wave constituent. When the wave distribution function should be recovered (Storey and Lefeuvre, 1974), then the appearance of the ion-type polarization in the electron whistler frequency band is the sign of a failure, indicating that the averaging time should be increased in order to reduce the influence of the interference cross terms, if possible. However, the truncation of the averaging time can also be useful: the field structure near the reflecting regions can be recovered and the information about the phase correlations between the constituents of the compound signals can be gathered.

Finally, let us mention that one of the possible manifestation of magnetic field interference patterns with ion-type polarization registered on the Earth's surface in the electron whistler frequency band has been recently reported by Manninen and Turunen $(1999,2000)$. The authors have demonstrated that the preferential sense of magnetic field polarization for the power line harmonics radiation (PLHR) is of iontype, while for the usual whistlers it is of the electron-type. This difference can be connected with the spatial structure of the magnetic field of the usual whistlers' transient signals and with the probable steady-state nature of the field structure of the PLHR emissions near the Earth's surface. The complete clarification of the curious physical phenomena described in this paper will be possible after the publication of the avail- 
able experimental data.

\section{Appendix A The probability of registration of the inverse sense of polarization in the field pattern of two noncorrelated sources}

Let us study the characteristics of the field interference pattern of two waves of relative amplitudes and phases $\sigma_{j}$ and $\delta_{j}$, respectively (see also notations in the text after Eq. 3). One can consider the total time interval $\left[0, T_{d}\right]$ of wave field registration as a large set of short intervals of equal duration $T_{k}$ (for simplicity) so that the variation over the intervals $T_{k}$ of the parameters $\left(\sigma_{1}, \sigma_{2}, \delta_{1}, \delta_{2}\right)_{k}$, characteristic of the two wave constituents, can be described with the help of a distribution function $F\left(\sigma_{1}, \sigma_{2}, \delta_{1}, \delta_{2}\right)$. To calculate the fraction of time $d_{2}$ during which the property ' $G$ ' of the field is verified in the registered interference pattern (here, ' $G$ ' is the sense of polarization which is opposite to that of the second constituent with subscript ' 2 '), one can directly add the corresponding time intervals $T_{k}(G)$ scattered over $\left[0, T_{d}\right]$, i.e.

$d_{2}=\frac{1}{T_{d}} \sum T_{k}(G)$.

The value $d_{2}$ is actually the probability to register inversely polarized fields when compared to the sense of polarization of the second wave constituent; using the distribution function $F(\boldsymbol{\sigma}, \boldsymbol{\delta}) \equiv F\left(\sigma_{1}, \sigma_{2}, \delta_{1}, \delta_{2}\right)$, one can write

$d_{2} \equiv \int_{G} d \sigma d \delta F(\sigma, \delta)$,

where $\sigma \equiv\left(\sigma_{1}, \sigma_{2}\right)$ and $\delta \equiv\left(\delta_{1}, \delta_{2}\right) ; G$ is the region of $(\boldsymbol{\sigma}, \boldsymbol{\delta})=\left(\sigma_{1}, \sigma_{2}, \delta_{1}, \delta_{2}\right)$ values where the inverse sense of polarization is registered (similar to the domains $G_{-}$and $G_{+}$defined in Sect. 2 of the text). The distribution function $F(\boldsymbol{\sigma}, \boldsymbol{\delta})$ of the wave field parameters should be chosen in accordance with the probable nature of the fields' sources or derived directly from the observation of the wave fields.

Let us calculate the mean value of the polarization parameter $P$ (see also Eq. (2) in the text) according to the definition

$P_{G} \equiv \int_{G} d \sigma d \delta F(\sigma, \delta) P(\sigma, \delta)$

and use the notation $\widehat{P}$ for the value of $P$ integrated over the total domain of $(\boldsymbol{\sigma}, \boldsymbol{\delta})$ values admissible for the investigated process; $\widehat{P}$ should coincide with the integral $\left(\int P d t\right) / T_{d}$ over the total time interval $T_{d}$.

Let us consider, as a possible example, the distribution function $F(\boldsymbol{\sigma}, \boldsymbol{\delta}) \equiv F_{1}\left(\sigma_{1}, \delta_{1}\right) F_{2}\left(\sigma_{2}, \delta_{2}\right)$ corresponding to the system of two independent wave field constituents; moreover, let us assume for simplicity that $F_{j}\left(\sigma_{j}, \delta_{j}\right) \equiv$ $\mathcal{F}_{j}\left(\sigma_{j}\right) \Phi_{j}\left(\delta_{j}\right)$, which is the case when the amplitude $\sigma_{j}$ of the constituent $j$ does not depend on its phase $\delta_{j}$. Finally, considering that phases are uniformly distributed, one can write that $F_{j}\left(\sigma_{j}, \delta_{j}\right)=\mathcal{F}_{j}\left(\sigma_{j}\right) / 2 \pi$. Using the so-called
Raleigh distribution function $\mathcal{F}_{j}\left(\sigma_{j}\right)$ for the amplitudes of the constituents

$\mathcal{F}_{j}\left(\sigma_{j}\right)=\left(\sigma_{j} / \beta_{j}^{2}\right) \exp \left(-\sigma_{j}^{2} / 2 \beta_{j}^{2}\right)$,

$\int_{0}^{\infty} d \sigma_{j} \mathcal{F}_{j}\left(\sigma_{j}\right)=1$,

full analytical calculations can be performed. In order to estimate the fraction of time $d_{2}$ when the inverse sense of polarization can appear, one needs to integrate the distribution function $F(\sigma, \delta)$ over the corresponding region $G$

$d_{2} \equiv \int_{G} d \sigma d \varphi \int_{0}^{2 \pi} d \delta_{1} \int_{0}^{\infty} d \sigma_{1} \sigma_{1} \mathcal{F}_{1}\left(\sigma_{1}\right) \mathcal{F}_{2}\left(\sigma \sigma_{1}\right) / 4 \pi^{2}$,

with $\sigma=\sigma_{2} / \sigma_{1}$ and $\varphi=\delta_{2}-\delta_{1}+v$ (see also Eq. (6) in the text). The domain $G$ is defined by Eqs. (13) or (14) depending on the sign of $p_{1} p_{2}$; for the definition of $p_{1,2}$ and other parameters used in following formulas, the reader should refer to the Sect. 2 of the text. Finally, one can find (Lundin, 1985) that

$d_{2}=\left(1-R / \sqrt{1-r+R^{2}}\right) / 2$

with

$r=p_{1} p_{2} /\left|p_{12}\right|^{2}$,

$R=\left(\operatorname{sign}(r)\left|p_{1}\right|+\widetilde{\sigma}^{2}\left|p_{2}\right|\right) / 2 \widetilde{\sigma}\left|p_{12}\right|$,

$\tilde{\sigma}=\beta_{2} / \beta_{1}$.

When $p_{1} p_{2}>0$ (i.e. $\operatorname{sign}(r)=1$ ), one has $d_{2} \leq 1 / 2$ and the maximum value $\left(d_{2}\right)_{\max }=(1-\sqrt{r}) / 2$ is reached for $\tilde{\sigma}^{2}=p_{1} / p_{2}$. When $p_{1} p_{2}<0, d_{2}$ changes monotonically from 1 to 0 while $\widetilde{\sigma}$ traces the interval ranging from 0 to $\infty$. Let us mention that in the simple case of two linearly polarized waves $\left(p_{1}=p_{2}=0\right)$ with noncolinear polarization vectors in a plane orthogonal to the unit vector $\boldsymbol{n}$, both clockwise and counterclockwise senses of rotation of the total field can be equivalently registered depending on the mutual phase difference $\varphi$. When $\varphi$ is uniformly distributed over the interval $[0,2 \pi]$, one can suppose that $d_{2}=1 / 2$, which is in accordance with Eq. (A6).

Let us calculate the ratio $D_{2}$ of the intensity $W_{G}$ of fields with inverse sense of polarization to the total field intensity $\widehat{W}$ localized in the plane orthogonal to $\boldsymbol{n}$

$D_{2} \equiv \frac{W_{G}}{\widehat{W}}=\frac{\iint_{G} d \boldsymbol{\sigma} d \boldsymbol{\delta} F(\boldsymbol{\sigma}, \boldsymbol{\delta}) W(\boldsymbol{\sigma}, \boldsymbol{\delta})}{\int d \boldsymbol{\sigma} d \boldsymbol{\delta} F(\boldsymbol{\sigma}, \boldsymbol{\delta}) W(\boldsymbol{\sigma}, \boldsymbol{\delta})}$.

After cumbersome calculations one finds that

$D_{2}=\frac{1}{2}\left(1-\frac{R}{\sqrt{1-r+R^{2}}}-\frac{R(1-r)}{2\left(1-r+R^{2}\right)^{\frac{3}{2}}}\right)$,

where we used the relation $\operatorname{Re}\left(p_{12} b_{12}^{*}\right)=\left(b_{2} p_{1}+b_{1} p_{2}\right) / 2$. Indeed, one can write using the notations (11) that

$$
\begin{aligned}
& 2 \operatorname{Re}\left(p_{12} b_{12}^{*}\right)=(\boldsymbol{t}, \boldsymbol{p})(\boldsymbol{p}, \boldsymbol{q})+(\boldsymbol{s}, \boldsymbol{q})(\boldsymbol{q}, \boldsymbol{p}) \\
& \quad=(\boldsymbol{t}, \boldsymbol{q})(\boldsymbol{p}, \boldsymbol{p})+(\boldsymbol{s}, \boldsymbol{p})(\boldsymbol{q}, \boldsymbol{q}) \\
& \quad+([\boldsymbol{t}, \boldsymbol{p}],[\boldsymbol{p}, \boldsymbol{q}])+([\boldsymbol{s}, \boldsymbol{q}],[\boldsymbol{q}, \boldsymbol{p}]) .
\end{aligned}
$$


As

$[p, q]=-n\left(\left[a_{1}, a_{2}\right], n\right)$

and

$([\boldsymbol{t}, \boldsymbol{p}], \boldsymbol{n})=i\left(\boldsymbol{a}_{2}, \boldsymbol{n}\right)\left(\boldsymbol{a}_{1}, \boldsymbol{n}\right)-i\left(\boldsymbol{a}_{1}, \boldsymbol{a}_{2}^{*}\right)=([\boldsymbol{s}, \boldsymbol{q}], \boldsymbol{n})$,

the two last terms in Eq. (A10) cancel each other so that

$$
\begin{aligned}
& 2 \operatorname{Re}\left(p_{12} b_{12}^{*}\right)=(\boldsymbol{t}, \boldsymbol{q})(\boldsymbol{p}, \boldsymbol{p})+(\boldsymbol{s}, \boldsymbol{p})(\boldsymbol{q}, \boldsymbol{q}) \\
& \quad \equiv p_{1} b_{2}+p_{2} b_{1} .
\end{aligned}
$$

$D_{2}$ can also be written as a function of $d_{2}$ in a very simple way

$D_{2}=d_{2}^{2}\left(3-2 d_{2}\right)$.

Then, the obvious relation $D_{2} \leq d_{2}$ (in the case when $p_{1} p_{2}>0$ so that $d_{2} \leq 1 / 2$ ) means that the relative intensity of the field recorded with the inverse sense of polarization is less than the relative time duration of its registration.

Calculations of $P_{G}$ and $C P L_{G}=P_{G} / W_{G}$ lead to (see also Eqs. (4), (7) and (15) in the text)

$$
\begin{aligned}
& P_{G}=-s_{2} 2 \beta_{1}^{2} \widetilde{\sigma}\left|p_{12}\right| \gamma d_{2}^{2}, \gamma \equiv \sqrt{1-r+R^{2}}, \\
& W_{G}=\beta_{1}^{2}\left(b_{2} \widetilde{\sigma}^{2}+b_{1}\right) d_{2}^{2}\left(3-2 d_{2}\right),
\end{aligned}
$$

and

$$
\left|C P L_{G}\right|=\left(\frac{2 \widetilde{\sigma}\left|p_{12}\right|}{b_{1}+\widetilde{\sigma}^{2} b_{2}}\right)\left(\frac{\gamma}{3-2 d_{2}}\right) .
$$

The mean value of the circular polarization level $\widehat{C P L}$ over the total domain of $(\boldsymbol{\sigma}, \boldsymbol{\delta})$ values is (see also Eq. (B8) of Appendix B):

$$
\widehat{C P L}=\frac{p_{1}+\widetilde{\sigma}^{2} p_{2}}{b_{1}+\widetilde{\sigma}^{2} b_{2}} .
$$

\section{Appendix B The spectral matrix approach}

Writing the wave field $\boldsymbol{K}(t)$ as a sum of several quasimonochromatic plane wave field constituents with constant complex polarization vectors $\boldsymbol{a}_{m}$ and complex amplitudes $h_{m}(t)$, namely

$$
\begin{aligned}
\boldsymbol{K}(t) & \equiv \frac{1}{2} \boldsymbol{H}(t) \exp (-i \omega t)+c . c . \\
& \equiv \frac{1}{2} \sum_{m} \boldsymbol{a}_{m} h_{m}(t) \exp (-i \omega t)+c . c .,
\end{aligned}
$$

one can define the spectral matrix (also called "coherence matrix" (Born and Wolf, 1959), "polarization tensor" (Landau and Lifshitz, 1960), or "Gram's matrix" (Ghantmakher, 1966) in mathematics) as

$$
S_{i j} \equiv\left(H_{i}, H_{j}\right) \equiv \frac{1}{T} \int_{0}^{T} H_{i}(t) H_{j}^{*}(t) d t .
$$

The elements of the matrix $S_{i j}$ are the scalar products of the functions $H_{i}(t)$ which are the components of the wave field vector $\boldsymbol{H}(t)(\mathrm{B} 1)$; the notation $\left(^{*}\right)$ corresponds to the complex conjugate value. Let us mention that for the case when only the amplitudes of the field components, i.e. $\left|H_{i}(t)\right|$, are registered (and not the phases), the corresponding spectral matrix can also be useful for the analysis of the wave field structure (Lefeuvre, 1977).

The subsequent conclusions about the field structure are based, typically, on the analysis of the eigen values of the Hermitian matrix $S_{i j}$ (B2) (Olson and Samson, 1979; Means, 1972). The existence of a single dominating eigen value is usually interpreted as the case when a nearly single plane wave is registered, even if this is actually not proved. Other cases need more complex interpretations (Voyevudskiy and Lundin, 1984). The properties of the spectral matrix under consideration depend strongly on the value of the averaging time $T$ chosen for data processing (see Eq. B2), on the time intervals between arriving quasi-monochromatic wave packets in actual signals, on the duration of these signals, as well as on the "inherent phase correlation time" between them. In general, the smaller the averaging time is, the more a single eigen value can dominate the others. The most complicated analysis includes the determination of the waves' distribution function under the assumption that the registered signal consists of noncorrelated spectral plane wave constituents (proportional to $\left|h_{m}\right|^{2}$ ) with linear polarization vectors $\boldsymbol{a}_{m}$ (Storey and Lefeuvre, 1974).

In application to the surrounding Earth's plasma, one can consider the case when large sets of wave packets arriving from different sources are registered. When the total duration $T_{d}$ of the registration is very long, one can assume that the phases of the arriving wave packets are distributed uniformly over the interval $[0,2 \pi]$, so that, as a result, one can present the spectral matrix of noncorrelated wave constituents numerated by $m$ as

$S_{i j}=\sum_{m}\left(a_{m}\right)_{i}\left(a_{m}^{*}\right)_{j} \lim _{T_{d} \rightarrow \infty} \frac{1}{T_{d}} \int_{0}^{T_{d}}\left|h_{m}(t)\right|^{2} d t$.

Under this assumption an algorithm of reconstruction of the so-called wave distribution function was developed by Storey and Lefeuvre (1974).

However, let us consider another approach to the processing of the multi-component wave field data; indeed, let us split the total registration time duration $T_{d}=\sum_{k} T_{k}$ into a large set of short intervals $T_{k}$ comparable with the delay times between arriving wave packets and their durations (these characteristic time scales of the process under consideration are not known in advance), so that one can assume that the amplitudes and the phases of the constituents are nearly constant during $T_{k}$ (then, a single eigen value dominates for the spectral matrix corresponding to $T_{k}$ ). Within some intervals $T_{k}(G)$ distributed over $\left[0, T_{d}\right]$, the spectral matrix can reveal rather unusual peculiarities of the wave field polarization (in comparison with plane wave polarization, for example). The spectral matrix $S_{i j}^{G}$, corresponding to 
the selected set of registration intervals $T_{k}(G)$, where the registered field reveals some special property labelled ' $G^{\prime}$, can be calculated in two ways: the first one consists in summing the integrals $S_{i j}^{G}(k)$ as follows

$S_{i j}^{G}=\sum_{k(G)} S_{i j}^{G}(k)$,

$S_{i j}^{G}(k) \equiv\left(H_{i}, H_{j}\right) \equiv \frac{1}{T_{k}(G)} \int_{T_{k}(G)} H_{i}(t) H_{j}^{*}(t) d t$,

where $k(G)$ represents the set of intervals $T_{k}(G)$ where the property ${ }^{\prime} G^{\prime}$ is realized. The second way consists in using the distribution functions of the parameters of the wave constituents $\boldsymbol{a}_{m} h_{m}(t)$ (see Eqs. (A3) and (A4), for example), which are supposed to be known beforehand. The probability of observing fields with the property ' $G^{\prime}$ can be found by calculating the ratio of the total time interval $T_{G}=\sum T_{k}(G)$ when ' $G^{\prime}$ is realized (for example, ' $G$ ' can represent the iontype polarization of the field in the electron whistler frequency band) to the total registration time $T_{d}$; one can also calculate integrals similar to Eq. (A3) over the domain $G$ of the wave constituents' parameters where the property ${ }^{\prime} G^{\prime}$ is realized. This mathematical approach can be used to estimate the polarization parameter $P_{G}$, the field intensity $W_{G}$ and the level of circular polarization $C P L_{G}$ (see Eqs. (A13), (A14) and (A15), respectively), corresponding to the regime of registration of the wave field when the property ${ }^{\prime} G^{\prime}$ is realized.

The polarization parameters $P$ and $W$ (see Eqs. (2) and (3) in the text) can be expressed through the elements of the corresponding matrix $S_{i j}$ (B2) by taking into account the following general relations

$S_{i j} \equiv R_{i j}+i J_{i j}=R_{i j}-e_{i j k} \varkappa_{k}$,

$\varkappa_{k} \equiv-\frac{1}{2} e_{k m n} S_{m n}, \quad e_{123}=1$,

$e_{k m n}=-e_{m k n}=-e_{k n m}$,

where $R_{i j}$ and $J_{i j}$ are the real and the imaginary parts of the matrix elements (the summation over repeated indexes is implied in Eq. B5). Then, the polarization parameter $P$ indicating the sense of field rotation is

$P=(\varkappa, n)=-J_{12}^{A}$,

where the $2 \mathrm{D}$ matrix $S_{i j}^{A} \equiv\left(S_{i j}\right)_{i, j \leq 2} \equiv R_{i j}^{A}+i J_{i j}^{A}$ is the main minor $(i, j \leq 2)$ of the $3 \mathrm{D}$ matrix $S_{i j}$ calculated in the coordinate system where the third axis is directed along the vector $\boldsymbol{n}$ ( $S_{i j}^{A}$ can be directly calculated using only two components of the field on the "oriented plane" of a twodimensional antenna $A$ orthogonal to $\boldsymbol{n}$ ). In the same manner one obtains the field intensity $W$ localized in the plane orthogonal to $\boldsymbol{n}$ as

$W=\frac{1}{2}\left(S_{11}^{A}+S_{22}^{A}\right)$,

so that the circular polarization level $C P L$ is expressed as

$C P L=\frac{P}{W}=-\frac{2 J_{12}^{A}}{\left(S_{11}^{A}+S_{22}^{A}\right)}$.
For the right-hand (with respect to $\boldsymbol{n}$ ) circularly polarized field one obtains $C P L=1$.

At last, let us briefly prove the known mathematical result that, in the case when the spectral matrix $S_{i j}$ (B2) has a single, nonvanishing eigen value, $\lambda=\sum S_{\alpha \alpha}$, the wave field (B1) can be expressed as

$$
\begin{aligned}
\boldsymbol{K}(t) & \equiv \frac{1}{2} \boldsymbol{H}(t) \exp (-i \omega t)+c . c . \\
& =\frac{1}{2} \boldsymbol{a} h(t) \exp (-i \omega t)+c . c .,
\end{aligned}
$$

where the constant polarization vector $\boldsymbol{a}$ is the eigen vector of the spectral matrix Eq. (B2). However, let us mention that the polarization vector $\boldsymbol{a}$ can be, in principle, crucially different from the plane wave polarization vector of some plasma mode, especially when considering the registration of steady-state interference patterns of different plane waves during the averaging time duration $T_{d}$.

For the single, nonvanishing eigen value $\lambda$, the so-called functions of coherence $\Gamma_{\alpha \beta}$ satisfy the following conditions for any $\alpha$ and $\beta$ (hereafter, the summation over the repeated indexes $\alpha$ and $\beta$ is not implied)

$\Gamma_{\alpha \beta} \equiv \frac{\left|S_{\alpha \beta}\right|^{2}}{S_{\alpha \alpha} S_{\beta \beta}}=1$.

Using scalar products of the functions $H_{\alpha}(t)$ (B1) according to the definition (B2), the condition (B10) can also be written as

$$
\begin{aligned}
& \left(H_{\alpha}, H_{\alpha}\right)\left(H_{\beta}, H_{\beta}\right)=\left(H_{\alpha}, H_{\beta}\right)\left(H_{\beta}, H_{\alpha}\right) \\
& \quad=\left|\left(H_{\alpha}, H_{\beta}\right)\right|^{2} .
\end{aligned}
$$

In the general case, one can express $H_{\alpha}(t)$ through $H_{\beta}(t)$ as

$$
H_{\alpha}(t)=H_{\beta}(t) \frac{\left(H_{\alpha}, H_{\beta}\right)}{\left(H_{\beta}, H_{\beta}\right)}+\Psi(t),
$$

where the function $\Psi(t)$ is evidently orthogonal to $H_{\beta}(t)$, i.e. $\left(\Psi, H_{\beta}\right)=0$; then, one can obtain the identity

$\left(H_{\alpha}, H_{\alpha}\right) \equiv \frac{\left(H_{\alpha}, H_{\beta}\right)\left(H_{\beta}, H_{\alpha}\right)}{\left(H_{\beta}, H_{\beta}\right)}+(\Psi, \Psi)$.

If the condition (B11) is satisfied, one has $(\Psi, \Psi)=0$; then, the continuous function $\Psi(t)$ is equal to zero at any point of the interval $[0, T]$ where the scalar product (B2) is defined.

Thus, when the conditions (B10) are satisfied for any $\alpha$ and $\beta$, the corresponding Eqs. (B12) and (B13) are satisfied with $\Psi(t) \equiv 0$; then $\boldsymbol{H}(t)=h(t) \boldsymbol{a}$ and one can write

$S_{i j} a_{j}=a_{i}|\boldsymbol{a}|^{2} \frac{1}{T} \int_{0}^{T}|h(t)|^{2} d t \equiv \lambda a_{i}$,

where, for example, $h(t)=H_{1}(t)$ and the components of the constant vector $\boldsymbol{a}$ are selected as $a_{k}=\left(H_{k}, H_{1}\right) /\left(H_{1}, H_{1}\right)$, so that $\boldsymbol{a}$ is the eigen vector of the matrix $S_{i j}$ corresponding to the single eigen value $\lambda$; this proves the statement (B9). 
Acknowledgements. The authors acknowledge the Centre National de la Recherche Scientifique (CNRS, PICS 1310, France) and the Russian Academy of Sciences for their financial support. B. Lundin wishes also to thank the Institut Universitaire de France in Paris. The research described in this publication was also made possible by grant $\mathrm{N}^{\circ} 98-05-65025$ from the Russian Foundation for Basic Research and by grant PST.CLG.976896 from NATO.

Topical Editor G. Chanteur thanks two referees for their help in evaluating this paper.

\section{References}

Born, M. and Wolf, E.: Principles of Optics, Pergamon, New York, 1959.

Chen, F. F. and Boswell, R. W.: Helicons -the past decade, IEEE Trans. Plasma Sci., 25, 6, 1245-1257, 1997.

Ghantmakher, F. R.: The theory of matrixes, Moscow, Science, 1966.

Landau, L. D. and Lifshitz, E.: Theory of Field, Addison-Wesley, Reading, Mass., 1960.

Lefeuvre, F.: Analyse de champs d'ondes électromagnétiques aléatoires observés dans la magnétosphère à partir de la mesure simultanée de leurs six composantes, Thèse de Doctorat d'Etat, Université d'Orléans, Orléans, France, 1977.

Lefeuvre, F., Neubert, T., and Parrot, M.: Wave normal directions and wave distribution functions for ground-based transmitter signals observed on Geos, J. Geophys. Res., 87, 8, 6203-6217, 1982.

Lefeuvre, F., Rauch, J. L., Volkomiskaya, L. B., and Lundin, B.: Polarization of electromagnetic waves recorded onboard the AUREOL-3 satellite, in: Results of the ARCAD-3 project and the recent programmes in magnetospheric and ionospheric physics, CEPADEES-EDITIONS, 485-498, 1985.

Lester, M. and Smith, A. J.: Whistler duct structure and formation, Planet. Space. Sci., 28, 645-654, 1980.

Lundin, B.: The effects of the interaction of resonant particles with monochromatic whistler waves propagating obliquely to the ambient magnetic field under magnetospheric conditions, $\mathrm{PhD}$ thesis, Institute of Terrestrial Magnetism, Ionosphere and Radio Wave Propagation, IZMIRAN, Moscow, Russia, 1979.

Lundin, B.: The new approach to the identification of the waveguide propagation of the VLF waves: the investigation of the polarization features of the wave field pattern, in: The propagation of the radiowaves in the ionosphere, Moscow, IZMIRAN, 107-112, 1983 (in Russian).

Lundin, B.: On the polarization features of radiowaves reflected from the ionosphere, in: Investigations of structure and waves' properties of the near Earth's plasma, IZMIRAN, Moscow, (in Russian), 95-103, 1985.

Lundin, B., Chmyrev, V., Krafft C., and Matthieussent, G.: VLF emission produced by a rarefied electron beam during active experiments in the Earth ionosphere: Conditions of observation of the emission, J. Geophys. Res., 99, 8, 14 987-15003, 1994.

Lundin, B. V., Krafft, C., Matthieussent, G., Jiricek, F., Shmilauer, J., and Triska, P.: Excitation of VLF quasi-electrostatic oscillations in the ionospheric plasma, Ann. Geophysicae, 14, 27-32, 1996.

Manninen, J. and Turunen, T.: PLHR-triggered emissions - lefthanded polarized VLF waves?, Report on Comissions HG3 P.7 of XXVI GA URSI, Toronto, Canada, 1999.

Manninen, J. and Turunen, T.: About the polarization of PLHRtriggered VLF waves, Report on Section ST12 of XXV GA EGS, Nice, France, 2000.

Means, J. D.: Use of the three-dimensional covariance matrix in analyzing the polarization properties of plane waves, J. Geophys. Res., 77, 28, 5551-5559, 1972.

Moriyama, S., Okada, T., Hayakawa, M., and Iwai, A.: The effect of the Earth-ionosphere waveguide propagation on the polarization and arrival angles of whistlers, Proc. Res. Inst. Atmos., Nagoya Univ., 26, 17-25, 1979.

Olson, J. V. and Samson, J. C.: On the detection of the polarization states of Pc micropulsations, Geophys. Res. Lett., 6(5), 413-416, 1979.

Sawada, A., Nobata, T., Kishi, Y., Kimura, I., and Oya, H.: Electron density profile in the magnetosphere deduced from in situ electron density and wave normal directions of Omega signals observed by the Akebono (EXOS D) satellite, J. Geophys. Res., 98, 7, 11 267-11274, 1993.

Shafranov, V. D.: Electromagnetic Waves in Plasma, in Review of Plasma Physics, Consultants Bureau, N.Y., 3, 1-157, 1967.

Shoji, T., Sakawa, Y., Nakazawa, S., Kadota, K., and Sato, T.: Plasma production by helicon waves, Plasma Sources Sci. Techn., 2, 5-10, 1993.

Southwood, D. J. and Kivelson, M. G.: Relations between polarization and the structure of ULF waves in the magnetosphere, J. Geophys. Res., 89, 7, 5523-5529, 1984.

Storey, L. R. O. and Lefeuvre, F.: Theory for the interpretation of measurements of a random electromagnetic wave field in space, Space Res., 14, 381-386, 1974.

Voyevudskiy, V. P. and Lundin, B.: Determining the properties of the plasma and the VLF signal from the spectral matrix of the wave field detected in a region which is a source of magnetospheric noise, Geomagn. Aeron., 24, 47-51, 1984. 